\title{
FDG-Positron Emission Tomography/Computed Tomography of an Area of Interest
}

National Cancer Institute

\section{Source}

National Cancer Institute. FDG-Positron Emission Tomography/Computed Tomography of an Area of Interest. NCI Thesaurus. Code C137868.

Positron emission tomography combined with computed tomography of an anatomical area of interest with the use of the contrast agent fludeoxyglucose F 18 (18F-FDG) to enhance the image. 